\title{
Outcome of medical thoracoscopy in patients admitted to abbasia chest hospital
}

\author{
Hanan Mohamed Mohamed Rashad ${ }^{\mathrm{a}}$, Emad Eldeen Abdel Wahab Korraa ${ }^{\mathrm{b}}$, \\ Maryam Ali Abdelkader ${ }^{\mathrm{c}}$
}

\begin{abstract}
Introduction Medical thoracoscopy is an invasive procedure that may be performed by physicians for the investigation of exudative pleural effusion using local anesthesia, conscious sedation, and a rigid thoracoscope.
\end{abstract}

Objective The aim was to evaluate the outcome of thoracoscopy in Abbassia Chest Hospital, Cairo.

Patients and methods A retrospective study was conducted. The results of histopathological diagnosed was statistically analyzed.

Results A total of 123 patients were available for analysis. Their mean age was 58.63 (22-80) years, and 64 (52\%) were male. The mean duration of chest drain after procedure was 9.79 (3-41) days, and the duration of hospital stay was 24.54 (10-55) days.

Malignant histology was reported in 108 (87.8\%) patients, with $75(61 \%)$ cases of mesothelioma. In 108 (87.8\%) patients, pleurodesis was performed.

The incidence of complications among the patients was $12.2 \%$ of all patients. The most common complications were blocked drain and empyema; other complications included subcutaneous emphysema, drain dislodgement,

\section{Introduction}

Thoracoscopy was introduced more than 100 years ago by Hans-Christian Jacobaeus from Sweden and has become today the second most important endoscopic technique in respiratory medicine after bronchoscopy [1]. Inclusion criteria were as follows: (a) patients were eligible when they presented with exudative pleural effusion and had thoracocentesis as a nonconclusive diagnostic procedure and (b) patients should be able to give informed written consent. Exclusion criteria were as follows: (a) general contraindications to thoracoscopy, (b) lack of a pleural space owing to pleural adhesions, (c) inability to lay supine, (d) inability to tolerate a pneumothorax, (e) severe cardiac disease, (f) severe respiratory disease unrelated to the effusion, (g) severe coagulopathy (i.e. platelet count $<60000$ and the international normalized ratio $<1.2$.), (h) uncontrolled cough, and (i) hypoxemia not owing to pleural effusions [2]. Medical thoracoscopy involves passage of an endoscope through the chest wall and offers the clinician an access for direct visualization and taking pleural biopsies. It is a valuable diagnostic procedure, and in some cases, it can also be a therapeutic procedure [3]. Medical thoracoscopy is a relatively safe procedure with an extremely low mortality rate [4]. Indications of medical thoracoscopy include workup and diagnosis of and bronchopleural fistula; and the least common complications were respiratory failure and atrial fibrillation.

Conclusion Thoracoscopy is a minimally invasive procedure that allows visualization of the pleural space and intrathoracic structures. It enables taking pleural biopsies under direct vision, therapeutic drainage of effusions, and pleurodesis in one sitting.

Egypt J Bronchol 2019 13:81-86

(C) 2019 Egyptian Journal of Bronchology

Egyptian Journal of Bronchology 2019 13:81-86

Keywords: mesothelioma, pleural effusion, thoracoscopy outcome, tuberculous pleural effusion

${ }^{\mathrm{a}}$ M.B.B.Ch, ${ }^{\mathrm{b}}$ Professor of chest diseases, ${ }^{\mathrm{C}}$ Lecturer of chest diseases, Faculty of Medicine, Ain Shams University, Cairo, Egypt

Correspondence to Maryam Ali Abdelkader, Lecturer of chest diseases, Faculty of Medicine, Ain Shams University, Cairo, 11865, Egypt. Researcher ID: L-5655; Tel: 01006423390; e-mail: Maryam7676@yahoo.com

Received 8 August 2018 Accepted 18 November 2018

idiopathic pleural effusions, staging of lung cancer, staging for malignant pleural mesothelioma (MPM), site-directed biopsy of parietal pleura, and pleurodesis [5]. Major contraindications to thoracoscopy include the lack of a pleural space owing to pleural adhesions, inability to lay supine, inability to tolerate a pneumothorax, severe cardiac disease, severe respiratory disease unrelated to the effusion, and severe coagulopathy. Relative contraindications are uncontrolled cough and refractory hypoxemia that is not owing to pleural effusions [2].

\section{Patients and methods}

We performed a retrospective analysis of patients who underwent thoracoscopy for unexplained exudative pleural effusion at Abbassia Chest Hospital over a 12-month period (from October 2015 to September 2016).

Demographic details, procedure date, and histology reports were recorded from the procedure record book

This is an open access journal, and articles are distributed under the terms of the Creative Commons Attribution-NonCommercial-ShareAlike 4.0 License, which allows others to remix, tweak, and build upon the work non-commercially, as long as appropriate credit is given and the new creations are licensed under the identical terms. 
in our dedicated thoracoscopy room. Admission and discharge dates and date of death (if happened) were obtained from the hospital's patient administration system database.

Thoracoscopy was done using a thoracoscope, Richard wolf rigid thoracoscope (Germany). By the single entry technique under local anesthesia, followed by drainage of fluid present in the pleural space, and then induction of an ipsilateral pneumothorax to allow good visual exposure, suspicious areas instead of abnormal areas. Biopsies were collected in formalin solution for histopathological examination. Finally, at the end of the procedure, an intercostal tube under water seal was then inserted before wound closure to evacuate air and residual fluid. Chest tubes were removed when the amount of drained pleural fluid became less than $100 \mathrm{ml}$ daily. Chemical pleurodesis was done for proved malignant cases.

Data were coded and entered using the statistical package SPSS (statistical package for the social sciences; SPSS Inc., Chicago, Illinois, USA) version 24. Data were summarized using mean, standard deviation, median, minimum, and maximum in quantitative data and using frequency (count) and relative frequency (percentage) for categorical data. Comparisons between quantitative variables were done using the nonparametric Mann-Whitney test [6]. For comparing categorical data, $\chi^{2}$-test was performed. Exact test was used instead when the expected frequency is less than 5 [7]. Correlations between quantitative variables were done using Spearman's correlation coefficient [8]. $P$ values less than 0.05 were considered as statistically significant. $P$ values equal or less than 0.001 were considered as highly significant. $P$ values equal or more than 0.05 were considered as statistically insignificant.

\section{Results}

The study included 123 patients, comprising 59 (48\%) females and 64 (52\%) males; their mean age was 58.63 \pm 11.51 years. A total of 49 (39.8\%) patients were living in Cairo, 24 (19.7\%) patients were living in Helwan, 38 (30.8\%) patients were living in Qaliobia, and 12 (9.7\%) patients were living in other cities.

The mean duration of hospital stay was $24.54 \pm 8.46$ days, and the mean duration of post-thoracoscopic drainage with intercostal chest tube was $9.79 \pm 5.65$ days.

A total of $108(87.8 \%)$ patients were diagnosed as having malignant pleural effusion, whereas 15
(12.19\%) patients were diagnosed as having nonmalignant. The most common diagnosis was malignant mesothelioma in 75 (61\%) cases followed by mesothelioma versus metastatic carcinoma in 22 (17.9\%) cases. The least common diagnosis was tuberculous effusion in seven (5.7\%) patients. The most common cytology finding was epithelioid cells in $70(56.91 \%)$ cases, followed by mesothelial cells and lymphocytes in 12 (9.75\%) cases, and the least common cytology finding was multicystic cells in one case.

Of 123 patients, 15 (12.2\%) had complications. The most common complications were blocked drain and empyema; other complications included subcutaneous emphysema, drain dislodgement, and bronchopleural fistula, and the least common complications are respiratory failure and atrial fibrillation (Table 1).

In $93.49 \%(n=115)$ of the patients, a specific diagnosis was established. In $87.8 \%(n=108)$, pleurodesis was performed. Overall, $85.36 \%(n=105)$ were referred for oncotherapy, and $2.4 \%(n=3)$ of the patients died before referral to oncotherapy (mortality was owing to the main disease and not related to the procedure itself). in $5.7 \%(n=7)$, antituberculous treatment was given, and $2.4 \%(n=3)$ of the patients died. The indication for pleurodesis was malignant pleural exudate with subsequent need for thoracocentesis.

There were significant differences between patients with malignant and those with nonmalignant effusion regarding age. There were insignificant differences between patients with malignant and those with nonmalignant effusion regarding sex. There was a significant statistical difference between patients with malignant and those with nonmalignant effusion regarding age and living in Cairo (Table 2).

A positive correlation was found between duration of hospital stay and age.

There was a significant relation between complications and duration of drainage of intercostal tube (ICT). As

Table 1 Complications details

\begin{tabular}{lc}
\hline & Number of cases (\%) \\
\hline Complications details & \\
Blocked drain & $5(4.06)$ \\
Empyema & $4(3.25)$ \\
Subcutaneous emphysema & $3(2.44)$ \\
Drain dislodgement & $2(1.63)$ \\
Bronchopleural fistula & $2(1.63)$ \\
Respiratory failure & $1(0.8)$ \\
Atrial fibrillation & $1(0.8)$ \\
\hline
\end{tabular}


Table 2 Comparison between cases with malignant and non-malignant pleural effusion regarding the demographic data

\begin{tabular}{|c|c|c|c|}
\hline & Malignant $(n=108)[n(\%)]$ & Nonmalignant $(n=15)[n(\%)]$ & $P$ \\
\hline Age (years) (mean $\pm S D)$ & $59.46 \pm 11.51$ & $52.87 \pm 10.47$ & 0.038 \\
\hline \multicolumn{4}{|l|}{ Sex } \\
\hline Female & $52(48.1)$ & $7(46.67)$ & 0.914 \\
\hline Male & $56(51.9)$ & $8(53.33)$ & 0.914 \\
\hline \multicolumn{4}{|l|}{ Residence } \\
\hline Cairo & $39(36.1)$ & $10(66.67)$ & 0.024 \\
\hline Helwan & $23(21.3)$ & $1(6.67)$ & 0.299 \\
\hline Qaliobia & $35(32.4)$ & $3(20)$ & 0.389 \\
\hline Others & $11(10.2)$ & $1(6.67)$ & 1 \\
\hline
\end{tabular}

Table 3 Relation between complications and duration of drainage of ICT

\begin{tabular}{|c|c|c|c|}
\hline & \multicolumn{2}{|c|}{ Duration of ICT drainage (days) } & \multirow[t]{2}{*}{$P$ value } \\
\hline & Mean $\pm S D$ & Range & \\
\hline \multicolumn{4}{|c|}{ Complications } \\
\hline Yes & $21.07 \pm 8.58$ & $11.00-41.00$ & $<0.001$ \\
\hline No & $8.05 \pm 2.70$ & $1.00-17.00$ & \\
\hline
\end{tabular}

Table 4 Relation between mortality and duration of ICT drainage

\begin{tabular}{lccc}
\hline & \multicolumn{2}{c}{ Duration of ICT drainage (days) } & $P$ value \\
\cline { 2 - 3 } & Mean \pm SD & Range & \\
\hline Mortality & & & \\
Yes & $28.67 \pm 12.50$ & $16.00-41.00$ & $<0.001$ \\
No & $9.15 \pm 4.68$ & $1.00-32.00$ & \\
\hline
\end{tabular}

Table 5 Relation between diagnosis and complications

\begin{tabular}{lccc}
\hline & \multicolumn{2}{c}{ Diagnosis $[n(\%)]$} & $P$ value \\
\cline { 2 - 3 } & Malignant & Nonmalignant & \\
\hline Complications & & & \\
Yes & $13(12.3)$ & $2(11.1)$ & 1 \\
No & $93(87.7)$ & $16(88.9)$ & \\
\hline
\end{tabular}

in complicated patients, the mean duration of ICT drainage was $21.07 \pm 8.58$ days, whereas in noncomplicated patients, it was $8.05 \pm 2.70$ days (Table 3).

Moreover, a significant relation between mortality and duration of ICT drainage was found. Mean duration of ICT drainage in the dead patients was $28.67 \pm 12.50$ days, whereas in the rest of the patients, it was 9.15 \pm 4.68 days (Table 4).

There was no significant relation between diagnosis and complications (Table 5).

There was no significant relation between diagnosis and mortality (Table 6).

There was a highly significant statistical relation between malignant pleural effusion and residence in
Table 6 Relation between diagnosis and mortality

\begin{tabular}{cccc}
\hline & \multicolumn{2}{c}{ Diagnosis $[n(\%)]$} & $P$ value \\
\cline { 2 - 3 } & Malignant & Nonmalignant & \\
\hline Mortality & $3(2.8)$ & $0(0.0)$ & 1 \\
No & $103(97.2)$ & $18(100.0)$ & \\
\hline
\end{tabular}

Table 7 Relation between diagnosis and residence

\begin{tabular}{lcc}
\hline Residence & Malignant $(n=108)[n(\%)]$ & $P$ value \\
\hline Cairo & $39(36.1)$ & $<0.05$ \\
Helwan & $23(21.3)$ & $<0.001$ \\
Qaliobia & $35(32.4)$ & $<0.001$ \\
Others & $11(10.2)$ & $<0.001$ \\
\hline
\end{tabular}

Helwan, Qaliobia, and other cities rather than Cairo. However, there is a significant statistical relation between malignant pleural effusion and residence in Cairo (Table 7).

\section{Discussion}

Medical thoracoscopy is a valuable diagnostic procedure and, in some cases, can also be a therapeutic procedure. It involves passage of an endoscope through the chest wall and offers the clinician an access for direct visualization and taking pleural biopsies [3].

This study is a retrospective study of the outcome of medical thoracoscopy in Abbassia Chest Hospital retrospectively from October 2015 to September 2016.

The present study included 123 patients, and all of them had pleural effusion that has not been finally diagnosed after history, general and local examination, and investigations including thoracocentesis with physical, chemical, bacteriological, and cytological examination of the pleural fluid.

Of the studied 123 patients, 59 (48.0\%) were females and $64(52.0 \%)$ were males; the age of patients in the 
present study ranges from 22 to 80 years, with mean age of $58.63 \pm 11.51$ years.

Of these patients, $108(87.8 \%)$ were diagnosed as having malignant effusion, comprising 52 (48.1\%) females and $56(51.9 \%)$ males, and their mean age was $59.46 \pm 11.51$ years, whereas 15 (12.2\%) were diagnosed as having nonmalignant effusion patients, comprising seven (46.67\%) females and eight (53.33\%) males, and their mean age was $52.87 \pm 10.47$ years.

In this study, we studied 123 cases of pleural effusion. Their mean age was $58.63 \pm 11.51$ years. A total of 108 (87.81\%) cases were diagnosed as having malignant effusion, whereas 15 (12.19\%) were diagnosed as having nonmalignant effusion. There were significant differences between patients with malignant and those with nonmalignant effusion regarding age. This does not match with the results reported by Mabrouk et al. [9] who studied 103 cases of pleural effusion at Abbassia Chest hospital, and their mean age $55.37 \pm 13.95$ years. In their study, $87(84.4 \%)$ were diagnosed as having malignant effusion, whereas $16 \quad(15.6 \%)$ were diagnosed as having nonmalignant effusion. The present study result also does not matching with the results reported by Aleman et al. [10] who studied 346 patients with pleural effusion, where 263 patients had malignant effusion, whereas 83 patients had idiopathic effusion; there were insignificant differences between patients with malignant effusion and those with idiopathic effusion regarding age. The unmatched results with the two previous studies may be owing to difference in sample sizes between the studies.

There were insignificant differences in this study between patients with malignant and those with nonmalignant effusion regarding sex. This matches with the results reported by Mabrouk et al. [9] who studied 103 patients with pleural effusion at Abbasia Chest Hospital; $84.4 \%$ were diagnosed as having malignant effusion, whereas 16 (15.6\%) were diagnosed as having nonmalignant effusion. There was a nonsignificant statistical difference between patients with malignant and those with idiopathic effusion regarding sex. This also matches with the results reported by Aleman et al. [10] who studied 346 patients with pleural effusion, where 263 patients had malignant effusion and 83 patients had idiopathic effusion; there were insignificant differences between patients with malignant and those with idiopathic effusion regarding sex.

Regarding residence, in the current study, there was a highly significant statistical relation between malignant pleural effusion and residence in Helwan, Qaliobia and other cities rather than Cairo. However, there is a significant statistical relation between malignant pleural effusion and residence in Cairo. This result does not match with Mabrouk et al. [9], who studied 103 cases of pleural effusion at Abbassia Chest hospital and found a significant association between malignant effusion and living in Helwan only. This also does not match with Madkour et al. [11], who studied 3892 cases, where 2913 of them were environmentally exposed to asbestos and a control group of 979 with no history of exposure; they found that there is exposure response relationship between asbestos and MPM. This was an epidemiological and environmental study that was carried out in Shubra El-Kheima City, Greater Cairo. The unmatching may be owing to increased industrial regions and increased pollution rate in all cities of Egypt.

In the current study, the mortality ratio is $2.4 \%$ (mortality was owing to the main disease and not related to the procedure itself); the incidence of complications between the patients was $12.2 \%$. The most common complications were blocked drain (4.06\%) and empyema (3.25\%); other complications included subcutaneous emphysema (2.44\%), drain dislodgement (1.63\%) and bronchopleural fistula (1.63\%), and the least common complications are respiratory failure $(0.8 \%)$ and atrial fibrillation $(0.8 \%)$. This does not match with the results obtained by Michaud et al. [2]. Their study was conducted on 50 patients with unexplained pleural effusion referred for medical thoracoscopy at Abbassia Chest Hospital, and they showed that 10\% had minor complications: $4 \%$ had infection (cellulitis) at the drain site, $4 \%$ had surgical emphysema, and $2 \%$ had bronchopleural fistula. This also does not match with results of Ahmed et al. [12]. Their study was carried out at the chest department of Zagazig University Hospitals in the period from October 2014 to October 2015. It included 36 patients with undiagnosed pleural effusion. They showed that postthoracoscopic complications in their studied groups were surgical emphysema and prolonged air leak in three $(8.3 \%)$ patients, pain during procedure in two (5.6\%) patients, wound infection in two (5.6\%) patients, and dislodged tube in two (5.6\%) patients (high incidence of surgical emphysema and prolonged air leak). This does not match with the results obtained by Yousef et al. [13], who studied 42 patients with undiagnosed exudative pleural effusion at Chest Department, Faculty of Medicine, Beni-Suef University. They showed that only one patient of the total $42(2.38 \%)$ studied patients died 1 day after 
thoracoscopy, as the patient developed vomiting after the procedure. Refractory hypoxemia and acute respiratory distress syndrome were developed. Otherwise, three (7.14\%) patients of total 42 studied patients developed surgical emphysema. This unmatching in thethree 3 previous studies may be owing to the difference in sample sizes.

In this study, the most common cytology finding among patients with malignant effusion was epithelioid cells in 70 (56.91\%) cases, followed by mesothelial cells and lymphocytes in 12 (9.75\%) cases. However, inflammatory cells were the most common finding among patients with nonmalignant effusion. This does not match with Mabrouk et al. [9] Their study included 103 patients with undiagnosed pleural effusion who presented to Abbassia Chest Hospital retrospectively from January 2010 to November 2011 and prospectively from December 2011 to June 2012. They showed that atypical cells were the most common finding among patients with malignant effusion (21, $24 \%)$, whereas lymphocytes were the most common finding among patients with nonmalignant effusion. Lymphocytes were significantly associates with nonmalignant effusion than malignant effusion [8 (50\%) vs. 19 (21.8\%)]. Inflammatory cells and lymphocytes were significantly associated with nonmalignant effusion than malignant effusion [three $(18.7 \%)$ vs. one (1\%)]. The unmatching may be owing to availability of more accurate facilities in histopathological field that facilitates more specific evaluation of tissue biopsies.

The most common diagnosis in this study was malignant mesothelioma in 75 (61\%) cases followed by mesothelioma versus metastatic carcinoma in 22 (17.9\%) cases, metastatic carcinoma (8.9\%), and nonspecific pleurisy (NSP) (6.5\%). The least common diagnosis was tuberculous effusion in seven (5.7\%) patients. This matches with the results obtained by Michaud et al. [2]. Their study was conducted on 50 patients with unexplained pleural effusion referred for medical thoracoscopy at Abbassia Chest Hospital, and they found that the most common diagnosis of pleural diseases was malignant mesothelioma (epithelial type) in $30(60 \%)$ patients, followed by metastatic carcinoma in six (12\%) patients. Regarding other nonmalignant diagnosis, tuberculous pleurisy was found in one (2\%) patient, fibrotic pleurisy in one (2\%) patient, and two (4\%) other cases remained undiagnosed. This also matches with the results obtained by Mabrouk et al. [9]. Their study included 103 patients with undiagnosed pleural effusion who presented to Abbassia Chest hospital retrospectively from January 2010 to November 2011 and prospectively from December 2011 to June 2012. The most common diagnosis in their study was malignant mesothelioma in $61(59.5 \%)$ cases followed by metastatic carcinoma in 23 (22.3\%) cases, chronic NSP in seven (6.8\%) cases, tuberculous pleurisy in seven (6.8\%) cases, malignant lymphoma in three $(2.9 \%)$ cases, fibrotic pleurisy in one (1\%) case, and only one (1\%) case was still undiagnosed. This is also matches with the results obtained by Yousef et al. [13] They studied 42 patients with undiagnosed exudative pleural effusion at Chest Department, Faculty of Medicine, Beni-Suef University and found that of 42 patients, nine (21.4\%) patients were diagnosed with MPM, 10 (23.8\%) patients with metastatic adenocarcinoma primary from breast, lung, and liver, one (2.3\%) patient with undifferentiated carcinoma, five (11.9\%) patients with caseating tuberculous granuloma, nine (21.4\%) with NSP, and eight (19.04\%) with empyema. This also matches with Ahmed et al. [12]. Their study was carried out at the Chest Department of Zagazig University Hospitals in the period from October 2014 to October 2015. It included 36 patients with undiagnosed pleural effusion, and they showed the following: the histopathological yield of thoracoscopic pleural biopsies was as follows: 25 (69.4\%) malignant cases, two (5.6\%) patients with tuberculous pleuritis, one $(2.8 \%)$ patient diagnosed with empyema, and another one $(2.8 \%)$ with rheumatoid arthritis; moreover, there were seven (19.4\%) patients with nonspecific inflammation. Study limitations in this research include missing detailed data about the sclerosing material and its success rate, and also the cause of death in patients with malignant pleural effusion, and this is may be contributed to being a retrospective study.

\section{Conclusion}

Medical thoracoscopy is a minimally invasive procedure that allows visualization of the pleural space and intrathoracic structures. Medical thoracoscopy enables taking pleural biopsies under direct vision and performing therapeutic drainage of pleural effusion. Persistent and recurrent exudative pleural effusions become common, and thoracocentesis does not give a definitive diagnosis in many patients. Therefore, thoracoscopy today remains the gold standard for these cases.

\section{Financial support and sponsorship}

Nil. 


\section{Conflicts of interest}

There are no conflicts of interest.

\section{References}

1 Seijo LM, Sterman DH. Interventional pulmonology. N Engl J Med 2001; 344:740-749.

2 Michaud G, Berkowitz DM, Ernst A. Pleuroscopy for diagnosis and therapy for pleural effusions. Chest 2010; 138:1242.

3 Rodriguez-Panadero F, Janssen JP, Astoul P. Thoracoscopy: general overview and place in the diagnosis and management of pleural effusion. Eur Respir J 2006; 28:409.

4 Rahman NM, Ali NJ, Brown G. British Thoracic Society Pleural Disease Guideline Group. Local anesthetic thoraco-scopy: British Thoracic Society Pleural Disease Guideline 2010. Thorax 2010; 65 (Suppl 2):ii54-ii60.

5 Jacobaeus HC. The cauterization of adhesions in artificial pneumothorax treatment of pulmonary tuberculosis under thoracoscopic control. Proc $R$ Soc Med 1923; 16:45-62.

6 Chan YH. Biostatistics102: quantitative data - parametric \& nonparametric tests. Singapore Med J 2003a; 44:391-396.
7 Chan YH. Biostatistics 103: qualitative data - tests of independence. Singapore Med J 2003b; 44:498-503.

8 Chan YH. Biostatistics 104: correlational analysis. Singapore Med J 2003c; 44:614-619.

9 Mabrouk $\mathrm{H}$, Ashmawi SSA, Salem HM. Evaluation of medical thoracoscopy in diagnosis of unidentified exudative pleural effusion in Abbassia Chest Hospital fromJanuary 2010 to June 2012. 2013.

10 Aleman C, Sanchez L, Alegre J, Ruiz E, Vazquez A. Differentiating between malignant and idiopathic pleural effusions: the value of diagnostic procedures. Q J Med 2007; 100:351-359.

11 Madkour MT, El Bokhary MS, Awad Allah HI, Awad AA, Mahmoud HF. Environmental exposure to asbestos and the ex-posure-response relationship with mesothelioma. East Mediterr Health J 2009; 15:25-38.

12 Ahmed MM, Abdel Halim HA, Aziz ET, El-Shorbagy RM. Outcomes and complications of medical thoracoscopy in undiagnosed exudative pleural effusion. Egypt J Bronchol 2016; 10:93-99.

13 Yousef ARI, Morsi AF, El-Shabrawy M, EI Shahaat HA. The role of medical thoracoscopy in the diagnosis of exudative pleural effusion at the Chest Department of Zagazig University Hospitals. Egypt J Bronchol 2016; 10:225-231. 